\title{
Contents of Volume 71
}

Vol. 71, No. 1, pp. 1-122

Indrikis A. Krams, Ronalds Krams, Priit Jõers, Māris Munkevics, Giedrius Trakimas, Severi Luoto, Sarah Eichler, David M. Butler, Enno Merivee, Anne Must, Markus J. Rantala, Jorge Contreras-Garduño and Tatjana Krama

Developmental speed affects ecological stoichiometry and adult fat reserves in Drosophila melanogaster

Lorenzo Rugiero, Massimo Capula, Daniele Dendi, Fabio Petrozzi, Massimiliano Di Vittorio and Luca Luiselli

A 28-years-long monitoring study suggests heavy decline and habitat shifts in three populations of a Mediterranean tortoise species

Liyun Wang, Shaogang Li, Lele Xu, Yongqin Li, Huaxu Chen and Daohai Chen

MicroRNA expression profiles of the cuttlefish (Sepiella japonica) during embryonic development

Han Zhao, Yaping Gao, Qiang Jiang, Jinpeng Wang, Wenhao Liu, Zhihua Ju, Xiuge Wang, Xiaochao Wei, Yundong Gao and Jinming Huang

Molecular characterization of the ACSS2 gene involved in adaptation to hypoxia in high-altitude cattle breeds

Lenize Batista Calvão, Carina Kaory Sasahara de Paiva, Joás da Silva Brito, Ana Luisa Fares, Calebe Maia, Thaisa Sala Michelan, Luciano Fogaça de Assis Montag and Leandro Juen

Influence of biotic and abiotic factors on adult Odonata (Insecta) in Amazon streams 


\section{Yunjie Wang, Yajun Yin, Gangtie Zheng and Hongxiang Yao}

Driving mechanism of dragonfly's wing flapping pattern for liquid circulation inside wing

\section{Short Notes}

Lixia Zhang, Yongsun Sheng, Xiangyu Yuan, Xueting Zhong and Xiaohong Chen

A skeletochronological estimation of age structure of a population of the paddy frog, Fejervarya multistriata, from the central east of China

\section{Michael Seiter, Marie Christine Moser and Thomas Schwaha}

Mating behavior and spermatophore morphology of the whip scorpion Typopeltis dalyi Pocock, 1900 (Uropygi, Thelyphonida)

Vol. 71, No. 2, pp. 123-242

\section{Hayato Takada}

Timing of the evening emergence of Hilgendorf's tube-nosed bat (Murina hilgendorfi) in relation to roost type and season

\section{Abdullah Altunışık and Halit Eksilmez}

Age, growth and survival rate in two populations of Darevskia derjugini (Nikolsky, 1898) from different altitudes (Squamata: Sauria: Lacertidae)

\section{Maja Mohorović and Miha Krofel}

The scent world of cats: where to place a urine scent mark to increase signal persistence?

\section{Madeline A. Richards and Thomas Hesselberg}

No edge effect on quantity of prey captured in the forest-dwelling tetragnathid orb spider Metellina mengei

\section{Lixia Zhang, Yongsun Sheng, Xiangyu Yuan, Fei Yu, Xueting Zhong,} Jiahong Liao, Zhenhao Liu and Wei Chen

Proximate mechanisms responsible for random mating by size in the Himalayan toad Duttaphrynus himalayanus

\section{Shashwat Singh, Geetanjali Mishra and Omkar}

Mating status of coexisting conspecific individuals modulate reproduction in a ladybird, Menochilus sexmaculatus 
Auke-Florian Hiemstra, Liselotte Rambonnet, Barbara Gravendeel and Menno Schilthuizen

The effects of COVID-19 litter on animal life

\section{Short Note}

\section{Daniel Pincheira-Donoso}

Correlated evolution between herbivory and gastrointestinal tract in a prolific lizard adaptive radiation

Vol. 71, No. 3, pp. 243-348

Samuel G. Penny, Rachel L. White, Dawn M. Scott, Lynne MacTavish and Angelo P. Pernetta

No evidence that horn trimming affects white rhinoceros horn use during comfort behaviour and resource access

\section{Ying Jiang, Long Jin, Yi Qiang Fu and Wen Bo Liao}

Association of social group with both life-history traits and brain size in cooperatively breeding birds

\section{Rogelio Roberto Muñoz-Li and Abdiel Jover Capote}

Diversity and community structure of moss- and lichen-dwelling tardigrades (Tardigrada) along an altitudinal gradient in Cuba

\section{Noriko Iwai and Kiyomi Yasumiba}

Field variations in mass at metamorphosis in a stream frog, Odorrana splendida, and their relationship with the stream environment

Mayra Zamora-Espinoza, Juan Carlos López-Acosta and Eduardo Mendoza

Anthropogenic perturbation modifies interactions between mammals and fruits in a tropical forest of southern Mexico

Ajša Alagić, Miha Krofel, Marko Lazić and Anamarija Žagar Effects of biotic and abiotic stressors on asymmetries and head size in two sympatric lizard species 
Vol. 71, No. 4, pp. 349-426

Seyyed Saeed Hosseinian Yousefkhani

Conservation biology of the critically endangered salamander, Paradactylodon persicus gorganensis (Clergue-Gazeau \& Thorn, 1979) (Amphibia: Hynobiidae) in northeastern Iran

Guojun Liu, Zhenhua Guo, Di Liu, He Meng, Yuming Zheng, Xiuhua Zhao, Lihong Gu, Zhifeng Chen, Xingyong Chen, Manyu Li, Jinyan Sun, Zhigang Ma, Haijuan He, Xiaolong Yu and Fanghong Hu Does gut microbiota regulate brooding in geese?

Claudiane S. Ramalheira, Márcia M.M. Cabral, Vera M.F. da Silva and Fernando C.W. Rosas

Giant otter behaviour in a mega-hydroelectric reservoir, Central Amazon, Brazil

Alexander Csanády and Ladislav Mošanský

Sex and age differences in skull size in Myodes glareolus from Slovakia

Yang Ya-zuo, Han Chun-yan, Jia Ting, Wang Zheng-kun, Zhu Wan-long and Zhang Hao

Variations of body mass and thermogenesis properties in Eothenomys olitor during cold acclimatization 